Original Article Journal of Epilepsy Research pISSN 2233-6249 / eISSN 2233-625

Received December 8, 2020

Revised April 20, 2021

Accepted April 23, 2021

Corresponding author:

Ahmad Reza Dehpour, PharmD, PhD

Department of Pharmacology, School of

Medicine, Tehran University of Medical

Sciences, Tehran, P.O. Box 13145-784, Iran

Tel. +98 2188973652

Fax. +982166402569

E-mail; Dehpour@yahoo.com

\section{Effect of Lenalidomide on Pentylenetetrazole-Induced Clonic Seizure Threshold in Mice: A Role for N-Methyl-D-Aspartic Acid Receptor/Nitric Oxide Pathway}

\author{
Elaheh Asgari Dafe, MS ${ }^{1,2}$, Nastaran Rahimi, MS ${ }^{1,2}$, Nina Javadian, MD ${ }^{1,2}$, Pegah Dejban, MD², \\ Monika Komeili, MS ${ }^{1,2}$, Sepideh Modabberi, MS ${ }^{1,2}$, Mehdi Ghasemi, MD, MPH \\ Ahmad Reza Dehpour, PharmD, PhD ${ }^{1,2}$ \\ ${ }^{1}$ Experimental Medicine Research Center, Tehran University of Medical Sciences, Tehran; ${ }^{2}$ Department of \\ Pharmacology, Tehran University of Medical Sciences, Tehran, Iran; ${ }^{3}$ Division of Nephrology and Hypertension, Mayo \\ Clinic, Rochester, $N Y ;{ }^{4}$ Department of Neurology, University of Massachusetts School of Medicine, Worcester, MA, \\ $U S A$
}

Background and Purpose: Accumulating evidence suggest that lenalidomide, a structural analog of thalidomide, has neuro-modulatory and neuroprotective properties. In the present study, we investigated effects of acute administration of lenalidomide on clonic seizure threshold in mice induced by pentylenetetrazole (PTZ) and possible role of N-methyl-D-aspartic acid receptor (NMDAR) and nitric oxide (NO) pathway.

Methods: We have utilized a clonic model of seizure in NMRI mice induced by PTZ to evaluate the potential effect of lenalidomide on seizure threshold. Different doses of lenalidomide $(5,10,20$, and $50 \mathrm{mg} / \mathrm{kg}$, intraperitoneal [i.p.]) were administered 1 hour before PTZ. To evaluate probable role of NMDAR/NO signaling, the non-selective NO synthase inhibitor $L-N^{G}$-nitroarginine methyl ester (L-NAME; $10 \mathrm{mg} / \mathrm{kg}$, i.p.), neuronal NOS (nNOS) inhibitor 7-nitroindazole (7-NI; $30 \mathrm{mg} / \mathrm{kg}$, i.p.), selective inducible NOS inhibitor aminoguanidine (AG; $100 \mathrm{mg} / \mathrm{kg}$, i.p.), selective NMDAR antagonist MK-801 (0.01 mg/kg, i.p.), and selective NMDAR agonist D-serine (30 mg/kg, i.p.) were injected 15 minutes before lenalidomide.

Results: Lenalidomide at 10 and $20 \mathrm{mg} / \mathrm{kg}$ significantly elevated the PTZ-induced seizure thresholds. Interestingly, L-NAME (10 mg/kg, i.p), 7-NI (30 mg/kg, i.p), and AG (100 mg/kg, i.p) reversed the anticonvulsive effect of lenalidomide $(10 \mathrm{mg} / \mathrm{kg}$ ). Moreover, treatment with the NMDAR agonist D-serine (30 mg/kg, i.p.) did not alter the anticonvulsive properties of lenalidomide (10 mg/kg, i.p). However, the NMDAR antagonist MK-801 (0.01 mg/kg, i.p) significantly reversed the anticonvulsive effects of lenalidomide (10 $\mathrm{mg} / \mathrm{kg})$.

Conclusions: Our study demonstrated a role for the NMDAR/NO pathway in the anticonvulsive effects of lenalidomide on the PTZ-induced clonic seizures in mice. (2021;11:6-13)

Key words: Lenalidomide, Seizures, Pentylenetetrazole, N-methyl-D-aspartate receptors, Nitric oxide synthase, Mice

\section{Introduction}

Lenalidomide is a derivative of the chemotherapy drug thalidomide and is mainly used for treatment of myelodysplastic syndromes. ${ }^{1}$ This medication seems to be more potent than thalidomide and achieves therapeutic responses at lower doses in both visceral and nervous system disorders. ${ }^{2,3}$ The adverse effect profile of these medication are also different. For instance, thalidomide can result in peripheral neuropathy by its cumulative dose, whereas lenalidomide rarely exhibits such side effect. ${ }^{2,4}$ Lenalidomide, when orally administrated, can penetrate the cerebrospinal fluid and be used for the central nervous system (CNS) malignancies in children. ${ }^{5}$ Neuroprotective effects of lenalidomide have also been shown in preclinical studies on neurodegenerative diseases such as amyotrophic lateral sclerosis ${ }^{6}$ and Parkinson's disease. ${ }^{7}$ 
Accumulating evidence indicate an anticonvulsive property for thalidomide in different animal models of seizure such as pentylenetetrazole (PTZ)-induced clonic seizures or amygdala kindling in rodents. ${ }^{8-10}$ This raises the possibility that thalidomide derivates such as lenalidomide may also exert similar effects on seizure threshold. However, this issue has not been investigated so far.

Lenalidomide interplays with many neurotransmitters in the biological system, among which nitric oxide (NO) is an important example. $^{11}$ NO has been shown to mediate both the peripheral and central actions of lenalidomide. ${ }^{12,13}$ The role of NO in the CNS function, especially in modulating seizure threshold, has been well documented in the literature. ${ }^{14-17} \mathrm{~N}$-methyl D-aspartic acid (NMDA) receptors (NMDARs) are identified as excitatory synaptic transmission and play pivotal roles in the seizure pathogenesis, hypoxic brain damage, and other CNS diseases. ${ }^{18}$ Additionally, the NMDAR-mediated NO signaling contributes to pathogenesis of seizures and other cortical brain dysfunctions. ${ }^{19-21}$ We also recently demonstrated a role for NO pathway as an underlying mechanism in the anticonvulsive effects of thalidomide in PTZ-induced clonic seizures in mice. ${ }^{10,22}$ Therefore, the objective of the present study was to assess the effects of lenalidomide on PTZ-induced clonic seizure threshold in mice and investigate possible involvement of the nitrergic and glutaminergic pathways in this effect of lenalidomide.

\section{Methods}

\section{Chemicals}

Chemicals that were used in this study included PTZ, L- $N^{G}$-nitroarginine methyl ester (L-NAME; a non-specific NO synthase inhibitor), 7-nitroindazole (7-Nl; a selective neuronal NO synthase [nNOS] inhibitor), aminoguanidine (AG; a selective inducible NO synthase [iNOS] inhibitor), D-Serine (an agonist of NMDAR), and MK-801 (a selective NMDAR antagonist). All drugs were purchased from Sigma (St. Louis, MO, USA). All chemicals, except for PTZ 0.5\% (which was administered intravenously), were intraperitoneally (i.p.) injected. 7-NI and lenalidomide were suspended in dimethyl sulfoxide (DMSO) 1\%, while other chemicals were dissolved in normal saline.

\section{Animals}

Male NMRI mice weighing 26 $\pm 3 \mathrm{~g}$ were purchased from Department of Pharmacology, Tehran University of Medical Sciences. Animals were kept under 12-h light/12-h dark cycle in a temperature-controlled room $\left(22 \pm 2^{\circ} \mathrm{C}\right)$ with humidity of $55 \pm 2 \%$. Our experiment was performed according to the National Institutes of Health guide for the care and use of Laboratory animals (NIH Publications No. 8023, revised 1978). Also, the experiment protocol has been approved by the local Ethical Committee. Eight animals were used in each group, and each animal was used only once.

\section{Seizure induction by pentylenetetrazole}

To assess the threshold of clonic seizures, a dental needle (30-gauge) was inserted into the lateral vein of mice's tail and fixed by adhesive tape; thereafter, mouse was allowed to walk freely. Meanwhile, an infusion pump pushed the PTZ solution (0.5\%) into the tail vein at a constant speed of $1 \mathrm{~mL} / \mathrm{min}$ and continued until forelimb clonus followed by full clonus of the body (general clonus). The seizure threshold was calculated in $\mathrm{mg} / \mathrm{kg}$ PTZ based on the time needed to appearance of the general clonus of limbs, the body weight of the animal, and the rate of infusion and concentration of PTZ in the infusate: ${ }^{22-25}$ the seizure threshold $=$ [infusion duration $(\mathrm{min}) \times$ infusion rate $(\mathrm{mL} / \mathrm{min}) \times \mathrm{PTZ}$ concentration $(\mathrm{mg} / \mathrm{mL}) \times 1,000] /$ [weight of mouse $(\mathrm{g})$ ].

\section{Experiments}

Lenalidomide at different doses $(5,10,20$, and $50 \mathrm{mg} / \mathrm{kg}$, i.p.) was administrated 60 minutes before PTZ-induced clonic seizure threshold assessment. The effective dose of lenalidomide (10 mg/kg, i.p.) were used for subsequent experiments. Mice were injected the equal volume of the vehicle ( $1 \%$ aqueous solution of DMSO) in all experiments and considered as control group. Regarding the assessment of the time course of lenalidomide, effective dose of lenalidomide (10 mg/kg, i.p.) was administered 30,60 , or 120 minutes prior PTZ. To assess the role of NO pathway, mice in separate groups received an acute administration of L-NAME (10 mg/kg), 7-NI (30 mg/kg), and AG (100 mg/kg) 15 minutes before vehicle and lenalidomide ( $10 \mathrm{mg} / \mathrm{kg}$ ) administration or 75 minutes before PTZ administration. A single dose of MK-801 ( 0.05 mg/kg, i.p.) and D-serine (30 mg/kg, i.p.) was administered 15 minutes before vehicle and lenalidomide administration or 75 minutes before the administration of PTZ. This study was performed to investigate the role of NMDA receptors in the lenalidomide effects on the PTZ-induced clonic seizure threshold. The dose and time line for administration of these drugs were based on a pilot study and our previous experiments. ${ }^{10,17}$ 


\section{Statistical analysis}

Data were expressed as mean \pm standard deviation. In order to confirm the normal distribution of the data, we used homogeneity of variance test. Data were analyzed by one-way analysis of variance (ANOVA) followed by post hoc Tukey's test and $p<0.05$ was considered statistically significant.

\section{Results}

\section{Effect of lenalidomide and its time courses on seizure threshold}

Figure $1 \mathrm{~A}$ illustrates the effect of the different doses of lenalidomide $(5,10,20$, and $50 \mathrm{mg} / \mathrm{kg}$, i.p.) on the clonic seizure threshold induced by PTZ. Analyses revealed a significant anticonvulsive effect of lenalidomide at doses of $10 \mathrm{mg} / \mathrm{kg}(p<0.001)$ and $20 \mathrm{mg} / \mathrm{kg}$ $(p<0.01)$ in comparison with control group. Figure $1 \mathrm{~B}$ depicts the ef-
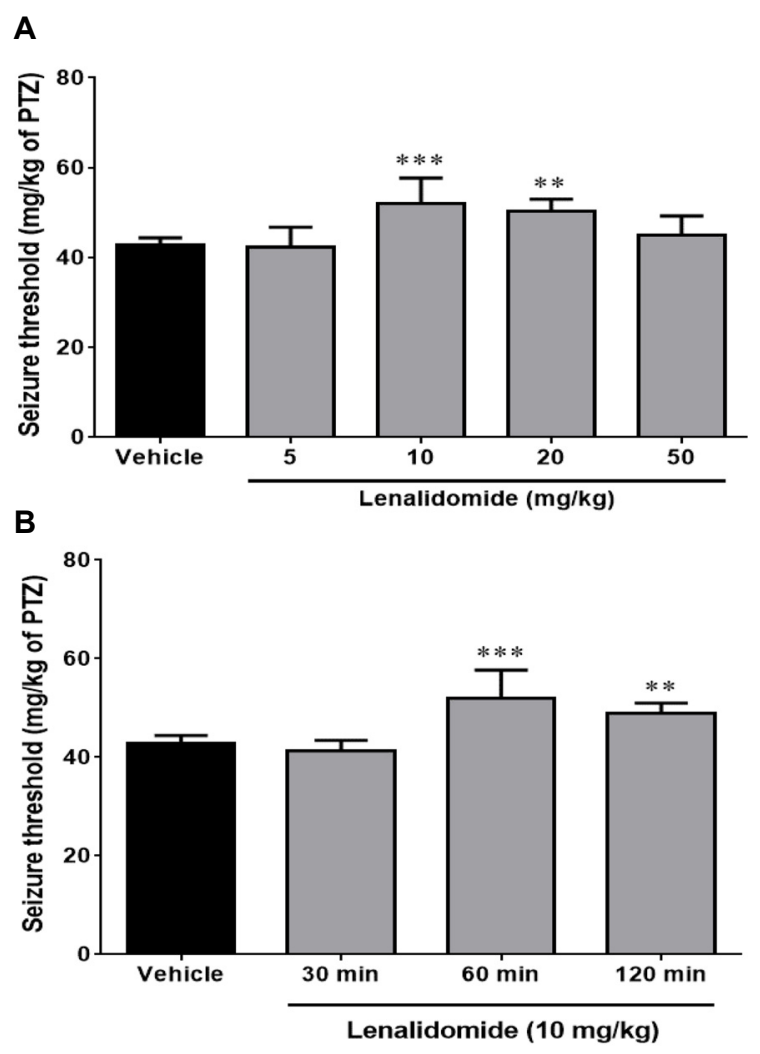

Figure 1. Effect of different doses of lenalidomide on pentylenetetrazole (PTZ)-induced seizures threshold (A). Time course of lenalidomide (10 mg/kg) in PTZ-induced clonic seizure (B). Data were analyzed by one-way analysis of variance followed by post hoc Tukey's test. Bars represent mean \pm standard deviation. ${ }^{* *} p<0.01$ and ${ }^{* * *} p<0.001$ compared with control group. fect of time course on the anticonvulsive activity of lenalidomide (10 $\mathrm{mg} / \mathrm{kg}$, i.p.). Lenalidomide did not change the seizure thresholds when it administered 30 minutes before the PTZ infusion, but it had a maximum anticonvulsive property 60 minutes after injection ( $p<$ 0.001 , compared with the control group).

A

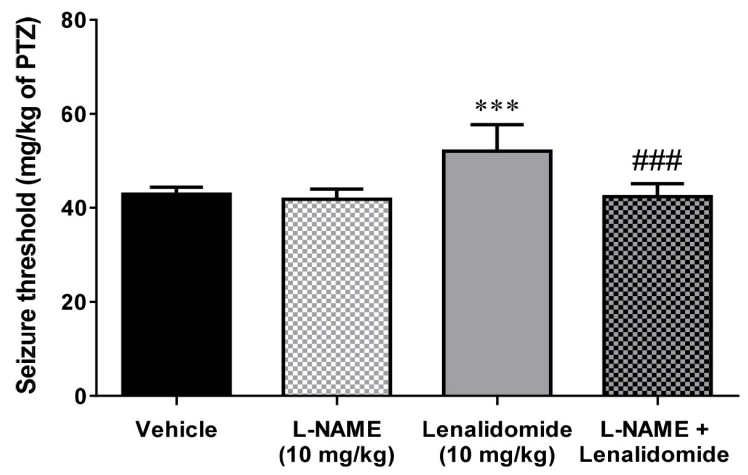

B

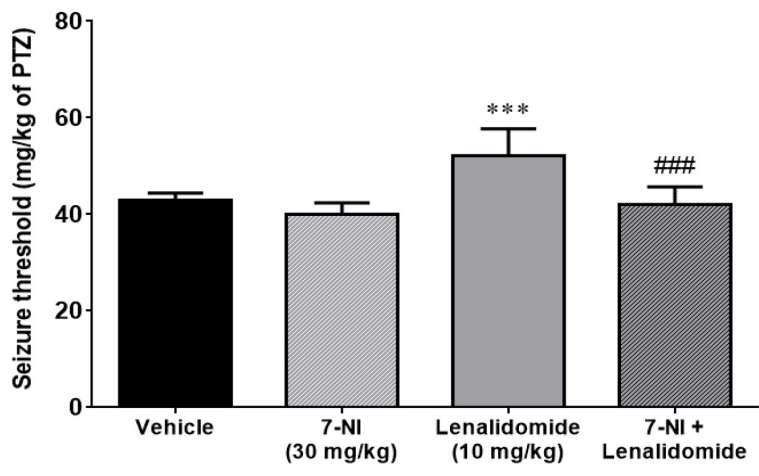

C

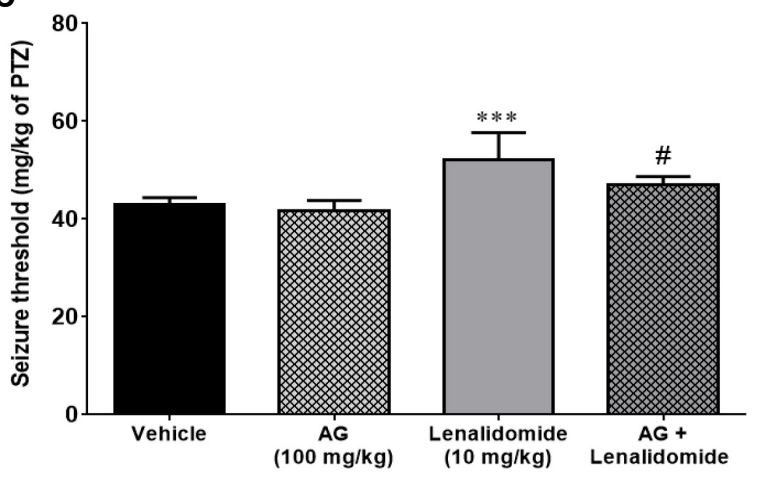

Figure 2. Effect of $L-N^{6}$-nitroarginine methyl ester (L-NAME; A), 7-nitroindazole (7-NI; B), and aminoguanidine (AG; C) on anticonvulsive effects of lenalidomide. Data were analyzed by one-way analysis of variance followed by post hoc Tukey's test. Bars represent mean \pm standard deviation. PTZ, pentylenetetrazole. ${ }^{* * *} p<0.001$ compared with the vehicle -treated group. ${ }^{*} p<0.05$ and $\ldots$ \# $p<0.001$ compared with lenalidomide-treated $(10 \mathrm{mg} / \mathrm{kg}$ ) group. Eight mice were used in each experimental group. 


\section{Effect of L-NAME on the anticonvulsive effects of lenalidomide}

Figure 2A demonstrates the effect of L-NAME injection, alone and 15 minutes before vehicle or lenalidomide (10 mg/kg, i.p), on the PTZ-induced clonic seizure threshold. Although L-NAME $(10 \mathrm{mg} / \mathrm{kg}$, i.p.) per se did not alter seizure threshold compared with the control group ( $p>0.05$ ), it prevented anticonvulsive effects of lenalidomide on seizure threshold $(p<0.001)$.

\section{Effects of 7-NI on the anticonvulsive effects of lenalidomide}

As shown in Figure 2B, the non-effective dose of 7-NI (30 mg/ $/ \mathrm{kg}$, i.p) did not affect the threshold of seizure when injected alone compared to the control group ( $p>0.05$ ). However, pretreatment with the same dose of 7-NI, 15 minutes before the administration of lenalidomide, markedly reduced the anticonvulsive influence of lenalidomide (10 mg/kg, i.p; $p<0.001$ ).

\section{Effect of AG on the anticonvulsive effects of lenalidomide}

As represented in Figure $2 \mathrm{C}$, administration of AG alone (100 $\mathrm{mg} / \mathrm{kg}$, i.p.) did not have any significant effect on seizure threshold compared to the control group. Pre-treatment with AG (100 mg/kg, i.p) 15 minutes before the administration of effective dose (10 $\mathrm{mg} / \mathrm{kg})$ of lenalidomide significantly $(p<0.05)$ reversed the anticonvulsive effect of lenalidomide in comparison to the lenalidomide-treated group.

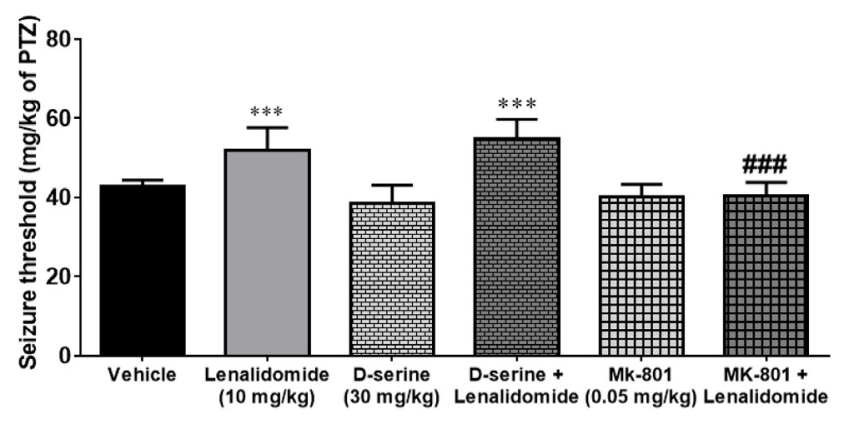

Figure 3. Effect of $\mathrm{N}$-methyl-D-aspartic acid receptors agonist (D-serine) and antagonist (MK-801) on anticonvulsive effects of lenalidomide. Data were analyzed by one-way analysis of variance followed by post hoc Tukey's test. Bars represent mean \pm standard deviation. PTZ, pentylenetetrazole. ${ }^{* *} p<0.001$ compared with the vehicle-treated group. ${ }^{\# \#} p<0.001$ compared with lenalidomidetreated $(10 \mathrm{mg} / \mathrm{kg}$ ) group. Eight mice were used in each experimental group.

\section{Effects of NMDAR agonist (D-serine) and antagonist (MK-801) on the anticonvulsive effects of lenalidomide}

Figure 3 illustrates effects of D-serine and MK-801 on the PTZ-induced clonic seizure thresholds in mice. Administration of the non-effective dose of D-serine (30 mg/kg, i.p) and MK-801 $(0.01 \mathrm{mg} / \mathrm{kg}$, i.p) per se was not associated with any significant alteration in the seizure threshold compared to control group. In addition, co-administration of D-serine (30 mg/kg, i.p) with lenalidomide did not change the effects of lenalidomide on the PTZ-induced clonic seizure thresholds compared to the lenalidomide (10 mg/kg, i.p) group ( $p>0.05)$. In contrast, combination of MK-801 $(0.01 \mathrm{mg} / \mathrm{kg})$ with lenalidomide (10 mg/kg, i.p) markedly decreased anticonvulsive effects of lenalidomide (10 mg/kg, i.p).

\section{Discussion}

The present study, for the first time, demonstrated that lenalidomide can markedly increase the PTZ-induced clonic seizure thresholds in mice. We also evaluated the possible involvement of both NMDAR and NO signaling pathways in the anticonvulsive effects of lenalidomide. To assess involvement of NO signaling, we used different NOS inhibitors including the non-selective NOS inhibitor L-NAME, the selective nNOS inhibitor 7-NI, and the selective iNOS inhibitor AG and evaluated their effects on anticonvulsive effects of lenalidomide. Pre-treatment with a per senon-effective dose of each of these NOS inhibitors in separate set of experiments significantly inhibited or attenuated the anticonvulsive effects of lenalidomide (10 $\mathrm{mg} / \mathrm{kg}$ ) on the PTZ-induced clonic seizure thresholds in mice. Overall, these data raise the possibility of NO contribution to the anticonvulsive effects of lenalidomide in this animal model of seizure. Additionally, to assess the involvement of NMDAR pathway, we used the selective NMDAR antagonist MK-801 and the selective NMDAR agonist D-serine. Our data showed that pre-treatment with a per se non-effective dose of MK-801 was able to inhibit the anticonvulsive effects of lenalidomide. This finding also suggests a role for NMDAR in the lenalidomide's effects on clonic seizure threshold. Based on the results of both NOS inhibitors and NMDAR antagonist in our study, the data can suggest the involvement of NMDAR and NO signaling in this effect of lenalidomide. It is well established that activation of NMDARs results in the activation of nNOS and related NO production and its downstream in the CNS. ${ }^{23-26}$ Accordingly, the results of the nNOS inhibitor 7-NI are in parallel with the NMDAR an- 
tagonist MK-801 in our study; thus, the NMDAR/nNOS could be a potential contributor to the lenalidomide effects on clonic seizure threshold. On the other hand, $A G$, which is a selective iNOS inhibitor (not a nNOS inhibitor), was also able to decrease the anticonvulsive effects of lenalidomide. AG is shown to be over 50 -fold more effective at inhibiting the enzymatic activity of iNOS than endothelial or neuronal NOS. ${ }^{27}$ This suggests that the source of NO in participating in the effects of lenalidomide on clonic seizure threshold in our study could be from either NMDAR/nNOS or iNOS activation.

Lenalidomide has been introduced as a derivative of thalidomide since 2004. It has been shown that clinical response to lenalidomide is maintained for a longer period of time in comparison with thalidomide in myelofibrosis patients. ${ }^{28}$ Moreover, lenalidomide exerts fewer side effects and has lower risks of peripheral neuropathy compared to thalidomide. ${ }^{28,29}$ Thalidomide's effects on brain electroencephalography has been reported in pregnant rodents, the electrical activity of the fetus brain increased with slow waves of high amplitude, ${ }^{30}$ however, after administration of this glutaminergic drug, electrophysiological recording of cat's brain was increased in the periods of slow waves and rapid eye movements, which were changed during sleep. ${ }^{31}$ For the first time, the antiepileptic effect of thalidomide was proposed by Palencia et al. ${ }^{8}$ in PTZ-provoked clonic seizures in rats. The same anticonvulsive effect again was reported later by the same group in another epilepsy model of amygdaloid kindling in rats. ${ }^{32}$ Consistently, in our recent studies, we found that thalidomide exerted anticonvulsive properties in PTZ-induced clonic seizure threshold in mice. ${ }^{10,22}$ One of the main findings of the present study was that the maximum anti-convulsive effect of lenalidomide was observed at a relatively low dose of lenalidomide (10 mg/ $\mathrm{kg}$ ). It is worth mentioning that the two major side effects of thalidomide and lenalidomide are teratogenicity and neuropathy in patients whose daily consumption reaches above $200 \mathrm{mg}$ for long periods. ${ }^{32}$

Lenalidomide also possesses anti-neuroinflammatory effects. ${ }^{33}$ Motor deficits reduction, dopaminergic fiber loss, microgliosis, and pro-inflammatory cytokine expression were elucidated as the mechanisms of lenalidomide effects on a mouse model of Parkinson's disease. $^{33}$ There are several case reports of anticonvulsive effects of thalidomide not only on inflammatory-induced epilepsy, known as Rasmussen encephalitis, but also on non-inflammatory refractory epileptic patients. ${ }^{6,32}$ Payandemehr at al. ${ }^{22}$ indicated that constitutive NO pathway mediates the anticonvulsive activity of thalidomide. Additionally, modulatory effects of thalidomide through iNOS signaling pathway were proposed in morphine dependency in mice. ${ }^{13}$ The role of nNOS in the thalidomide effects in immunomodulation has been reported in some studies. ${ }^{11,22}$ iNOS modulation has also been proposed as an underlying mechanism in immunomodulatory effects of lenalidomide. ${ }^{11}$ Furthermore, it was recently proposed that in mechanism of morphine dependency, lenalidomide could modulate NO level elevation in brain through activating phosphoinositide 3-kinase enzyme in brain, which leads to increasing cellular NO levels. ${ }^{13}$ Our current data are also in line with previous findings, which emphasizes a role for NO signaling in mediating the anticonvulsive effects of lenalidomide in mice.

The interaction of NO and NMDARs in the pathophysiology of seizure have been investigated. ${ }^{34,35}$ NMDARs are introduced as pivotal modulators of nNOS activity and NO release in the CNS. ${ }^{26,36}$ It was reported in clinical studies that felbamate, an NMDAR antagonist, demonstrated a therapeutic effect on controlling refractory partial seizures in humans. ${ }^{34,35}$ Although glutamate receptors are mainly known for their pro-convulsive properties, interaction of NMDA and $\mathrm{NO}$ in pathophysiology of both anticonvulsive and pro-convulsive effects of D-penicillamine on PTZ-induced clonic seizures in mice have been proposed. ${ }^{19}$ In studies evaluating the regulation of seizure susceptibility, it has been demonstrated that either NOS substrates or NO donors can exhibit both anticonvulsive $e^{37-39}$ or pro-convulsive ${ }^{40-43}$ effects in a variety of pre-clinical seizure models; this contradiction may be due to different experimental conditions (e.g., using different pharmacological tools to modify the NO signaling) in these studies. Accordingly, NO is demonstrated to contribute to both anticonvulsive and pro-convulsive effects of morphine on PTZ-induced clonic seizure in mice ${ }^{44,45}$ or lithium/pilocarpine-induced status epilepticus in rats. ${ }^{46}$ NOS inhibitors are also shown either to potentiate ${ }^{38,47-50}$ or to inhibit $^{42,47,50-52}$ experimentally induced seizures. This bimodal effects of NO on seizure susceptibility are in parallel with the fact that NO could exert either neuroprotective or neurotoxic effects in a variety of animal models of neurologic diseases. ${ }^{24,25}$ The levels of NO produced by different NOS isoforms could play an important role in this regard; at the lowest levels (usually nanomolar concentrations), normal physiologic role of NO is achieved primarily through reaction with ferrous hemes and related cyclic guanosine monophosphate-mediated signaling as well as with reactive radicals. ${ }^{53}$ However, excess levels of NO can interact with a variety of cytosolic, nonheme iron targets or convert into reactive nitrogen species leading to nitrosative, nitrative and oxidative modifications which are generally neurotoxic. ${ }^{23-25}$

Glutamate is the major excitatory neurotransmitter in the CNS and acts on ionotropic and metabotropic glutamate receptors, including 
NMDARs and $\alpha$-amino-3-hydroxy-5-methyl-4-isoxazole propionic acid receptor, which regulate neuronal excitability and synaptic plasticity. ${ }^{54,55}$ NO physiologically regulates activity of these receptors via S-nitrosylation, ${ }^{54,56}$ which is a reversible, covalent addition of NO group to a cysteine thiol/sulfhydryl to form an S-nitrosothiol derivative. ${ }^{57}$ NO S-nitrosylates GluN2A subunit of NMDAR, which inhibits NMDAR activity. ${ }^{56}$ This effect affords a negative feedback system for glutamate neurotransmission preventing excitotoxicity. ${ }^{56}$ $S$-Nitrosylation of NMDAR may induce a conformational change in the receptor protein that makes glutamate and $\mathrm{Zn}^{2+}$ bind more tightly to the receptor, causing the receptor to desensitize and, consequently, the ion channel to close. ${ }^{58}$ Therefore, one may suggest that the inhibition of NOS could suppress this regulatory effect of NO on glutamatergic receptors and increase the susceptibility for excitotoxicity and seizure progression. ${ }^{50}$

The dose of the NMDAR antagonist MK-801 $(0.05 \mathrm{mg} / \mathrm{kg})$ that we currently used did not individually affect the seizure thresholds, which is in consistent with our previous study. ${ }^{17}$ We found that combining lenalidomide with this non-effective dose of MK-801 reversed anticonvulsive effects of lenalidomide. Previously, we showed that MK-801 at higher doses such as $1 \mathrm{mg} / \mathrm{kg}$ inhibits clonic seizure thresholds induced by PTZ in mice, ${ }^{17}$ which is in agreement with the overall anticonvulsive property of NMDAR antagonists in pre-clinical studies. ${ }^{59-61}$ However, paradoxical pro-convulsive effects of MK-801 on seizure threshold have been also reported. ${ }^{38,62}$ The mechanisms underlying such paradoxical effects of MK-801 on seizure threshold remain to be elucidated. One possible explanation could be the differences in the distribution of NMDARs in the brain, as activation of NMDARs in certain brain regions can inhibit seizures. ${ }^{38}$ For instance, it was reported that direct injection of the NMDAR agonist NMDA into the corpus striatum $^{63}$ or substantia nigra pars compacta ${ }^{64}$ acted to release gamma aminobutyric acid (GABA) and dopamine, respectively, and inhibited pilocarpine-induced seizures in rodents. Thus, inhibition of NMDARs in these brain regions may be pro-convulsive. Clearly, more studies are needed to explore the mechanisms underlying paradoxical effects of NMDARs on seizure threshold in different brain regions.

The anticonvulsive effects of thalidomide have recently been proposed in animal models and human's studies. The majority of hypnotics and sedatives drugs have shown parallel anti-epileptogenic properties; thus, it seems logical that an analog of glutamic acid agent, thalidomide, and its derivative, lenalidomide, show the same anticonvulsive activity, as found in our present study. Of noted, there is no report of physical dependence to thalidomide or its derivative agent lenalido- mide, in contrast with other hypnotic and sedative drugs. Moreover, in suicidal attempts by these drugs, survival even after huge doses consumption has been reported. ${ }^{65,66}$ In the current study we used PTZ (a GABA receptor antagonist) to create a common chemically-induced clonic seizures in mice. The PTZ-induced seizures are categorized as a model of generalized seizure that produces a myoclonic seizure. ${ }^{67}$ When screening anticonvulsant candidates the PTZ model is a good tool for evaluating anti-seizure characteristics compared to a focal or partial seizure model. ${ }^{67}$ Beside assessing the time until the first whole body clonus event, some studies also assess time to the tonic-hind limb extension after PTZ injection, which may extend assessment for tonic seizures. ${ }^{68}$ However, it is noteworthy that in our study we only assessed the clonic seizures (and did not evaluate the time to tonic phase) after PTZ injection. In conclusion, our data indicated that acute administration of lenalidomide increases the PTZ-induced clonic seizure threshold in mice. Additionally, we demonstrated that this anticonvulsive activity of lenalidomide may be mediated by the NMDAR and NO pathway.

\section{Acknowledgements}

The study was supported by a Grant from Experimental Medicine Research Center, Tehran University of Medical Sciences, and a Grant (96002757) from the Iran National Science Foundation (INSF).

\section{Conflict of Interest}

The authors declare that they have no conflicts of interest.

\section{References}

1. Germing U, Schroeder T, Kaivers J, Kündgen A, Kobbe G, Gattermann $\mathrm{N}$. Novel therapies in low- and high-risk myelodysplastic syndrome. Expert Rev Hematol 2019;12:893-908.

2. Anderson KC. Lenalidomide and thalidomide: mechanisms of action--similarities and differences. Semin Hematol 2005;42(4 Suppl 4):S3-8.

3. Hideshima T, Raje N, Richardson PG, Anderson KC. A review of lenalidomide in combination with dexamethasone for the treatment of multiple myeloma. Ther Clin Risk Manag 2008;4:129-36.

4. Barth P, Giri S, Reagan JL, Olszewski AJ. Outcomes of lenalidomideor bortezomib-based regimens in older patients with plasma cell myeloma. Am J Hematol 2021;96:14-22.

5. Warren $\mathrm{KE}$, Goldman S, Pollack IF, et al. Phase I trial of lenalidomide in pediatric patients with recurrent, refractory, or progressive primary CNS tumors: pediatric brain tumor consortium study PBTC-018. J Clin Oncol 2011;29:324-9. 
6. Kiaei M, Petri S, Kipiani K, et al. Thalidomide and lenalidomide extend survival in a transgenic mouse model of amyotrophic lateral sclerosis. J Neurosci 2006;26:2467-73.

7. Valera E, Mante M, Anderson S, Rockenstein E, Masliah E. Lenalidomide reduces microglial activation and behavioral deficits in a transgenic model of Parkinson's disease. J Neuroinflammation 2015;12:93.

8. Palencia G, Calderon A, Sotelo J. Thalidomide inhibits pentylenetetrazole-induced seizures. J Neurol Sci 2007;258:128-31.

9. Palencia G, Rubio C, Custodio-Ramirez V, Paz C, Sotelo J. Strong anticonvulsant effect of thalidomide on amygdaloid kindling. Epilepsy Res 2011;95:263-9.

10. Pourshadi N, Rahimi $N$, Ghasemi $M$, et al. anticonvulsant effects of thalidomide on pentylenetetrazole-induced seizure in mice: a role for opioidergic and nitrergic transmissions. Epilepsy Res 2020;164:106362.

11. Ladizinski B, Shannon EJ, Sanchez MR, Levis WR. Thalidomide and analogues: potential for immunomodulation of inflammatory and neoplastic dermatologic disorders. J Drugs Dermatol 2010;9:814-26.

12. Vallet S, Palumbo A, Raje N, Boccadoro M, Anderson KC. Thalidomide and lenalidomide: mechanism-based potential drug combinations. Leuk Lymphoma 2008;49:1238-45.

13. Khan Ml, Momeny M, Ostadhadi S, et al. Thalidomide attenuates development of morphine dependence in mice by inhibiting PI3KJAkt and nitric oxide signaling pathways. Prog Neuropsychopharmacol Biol Psychiatry 2018;82:39-48.

14. Javadian N, Rahimi N, Javadi-Paydar M, Doustimotlagh AH, Dehpour AR. The modulatory effect of nitric oxide in pro- and anti-convulsive effects of vasopressin in PTZ-induced seizures threshold in mice. Epilepsy Res 2016;126:134-40.

15. Hassanipour M, Shirzadian A, Boojar MM, et al. Possible involvement of nitrergic and opioidergic systems in the modulatory effect of acute chloroquine treatment on pentylenetetrazol induced convulsions in mice. Brain Res Bull 2016;121:124-30.

16. Gholipour T, Ghasemi M, Riazi K, Ghaffarpour M, Dehpour AR. Seizure susceptibility alteration through $5-\mathrm{HT}(3)$ receptor: modulation by nitric oxide. Seizure 2010;19:17-22.

17. Ghasemi M, Shafaroodi $H$, Nazarbeiki S, et al. Inhibition of NMDA receptor/NO signaling blocked tolerance to the anticonvulsant effect of morphine on pentylenetetrazole-induced seizures in mice. Epilepsy Res 2010;91:39-48.

18. Mares $P$, Velísek L. N-methyl-D-aspartate (NMDA)-induced seizures in developing rats. Brain Res Dev Brain Res 1992;65:185-9.

19. Rahimi N, Sadeghzadeh $M$, Javadi-Paydar M, Heidary MR, Jazaeri $F$, Dehpour AR. Effects of D-penicillamine on pentylenetetrazole-induced seizures in mice: involvement of nitric oxide/NMDA pathways. Epilepsy Behav 2014;39:42-7.

20. Bahramnjead E, Kazemi Roodsari S, Rahimi N, Etemadi P, Aghaei I, Dehpour AR. Effects of modafinil on clonic seizure threshold induced by pentylenetetrazole in mice: involvement of glutamate, nitric oxide, GABA, and serotonin pathways. Neurochem Res 2018;43:2025-37.
21. Robello M, Amico C, Bucossi G, Cupello A, Rapallino MV, Thellung S. Nitric oxide and GABAA receptor function in the rat cerebral cortex and cerebellar granule cells. Neuroscience 1996;74:99-105.

22. Payandemehr B, Rahimian $R$, Gooshe $M$, et al. Nitric oxide mediates the anticonvulsant effects of thalidomide on pentylenetetrazole-induced clonic seizures in mice. Epilepsy Behav 2014;34:99-104.

23. Ghasemi M. Nitric oxide: a ntidepressant mechanisms and inflammation. Adv Pharmacol 2019;86:121-52.

24. Ghasemi M, Mayasi Y, Hannoun A, Eslami SM, Carandang R. Nitric oxide and mitochondrial function in neurological diseases. Neuroscience 2018; 376:48-71.

25. Ghasemi M, Claunch J, Niu K. Pathologic role of nitrergic neurotransmission in mood disorders. Prog Neurobiol 2019;173:54-87.

26. Leppik IE, Dreifuss FE, Pledger GW, et al. Felbamate for partial seizures: results of a controlled clinical trial. Neurology 1991;41:1785-9.

27. Corbett JA, MCDaniel ML. The use of aminoguanidine, a selective iNOS inhibitor, to evaluate the role of nitric oxide in the development of autoimmune diabetes. Methods 1996;10:21-30.

28. Jabbour E, Thomas D, Kantarjian H, et al. Comparison of thalidomide and lenalidomide as therapy for myelofibrosis. Blood 2011;118:899-902.

29. Luo J, Gagne JJ, Landon J, Avorn J, Kesselheim AS. Comparative effectiveness and safety of thalidomide and lenalidomide in patients with multiple myeloma in the United States of America: a population-based cohort study. Eur J Cancer 2017;70:22-33.

30. Bergstroem R, Bergström L, Putkonen $P$, Sainio $K$. The effects of thalidomide on the electrical activity of the brain in the intrauterine guinea-pig foetus. Med Exp Int J Exp Med 1964;11:119-27.

31. Kaitin Kl. Effects of thalidomide and pentobarbital on neuronal activity in the preoptic area during sleep and wakefulness in the cat. Psychopharmacology (Berl) 1985;85:47-50.

32. Palencia G, Martinez-Juarez IE, Calderon A, Artigas C, Sotelo J. Thalidomide for treatment of refractory epilepsy. Epilepsy Res 2010;92:253-7.

33. Valera E, Mante M, Anderson S, Rockenstein E, Masliah E. Lenalidomide reduces microglial activation and behavioral deficits in a transgenic model of Parkinson's disease. J Neuroinflammation 2015;12:93.

34. Noguchi T, Sano H, Shimazawa R, Tanatani A, Miyachi H, Hashimoto Y. Phenylhomophthalimide-type NOS inhibitors derived from thalidomide. Bioorg Med Chem Lett 2004;14:4141-5.

35. Ahmed MM, Arif M, Chikuma T, Kato T. Pentylenetetrazol-induced seizures affect the levels of prolyl oligopeptidase, thimet oligopeptidase and glial proteins in rat brain regions, and attenuation by MK-801 pretreatment. Neurochem Int 2005;47:248-59.

36. Ghasemi M, Dehpour AR. The NMDA receptor/nitric oxide pathway: a target for the therapeutic and toxic effects of lithium. Trends Pharmacol Sci 2011;32:420-34.

37. Buisson A, Lakhmeche N, Verrecchia C, Plotkine M, Boulu RG. Nitric oxide: an endogenous anticonvulsant substance. Neuroreport 1993;4: 444-6.

38. Starr MS, Starr BS. Paradoxical facilitation of pilocarpine-induced seizures 
in the mouse by MK-801 and the nitric oxide synthesis inhibitor L-NAME. Pharmacol Biochem Behav 1993:45:321-5.

39. Theard MA, Baughman VL, Wang Q, Pelligrino DA, Albrecht RF. The role of nitric oxide in modulating brain activity and blood flow during seizure. Neuroreport 1995;6:921-4.

40. Mülsch A, Busse R, Mordvintcev PI, et al. Nitric oxide promotes seizure activity in kainate-treated rats. Neuroreport 1994;5:2325-8.

41. Nidhi G, Balakrishnan S, Pandhi P. Role of nitric oxide in electroshock and pentylenetetrazole seizure threshold in rats. Methods Find Exp Clin Pharmacol 1999;21:609-12.

42. Osonoe K, Mori N, Suzuki K, Osonoe M. Antiepileptic effects of inhibitors of nitric oxide synthase examined in pentylenetetrazol-induced seizures in rats. Brain Res 1994;663:338-40.

43. Mollace V, Bagetta $G$, Nisticò $G$. Evidence that L-arginine possesses proconvulsant effects mediated through nitric oxide. Neuroreport 1991;2: 269-72.

44. Homayoun H, Khavandgar S, Namiranian K, Gaskari SA, Dehpour AR. The role of nitric oxide in anticonvulsant and proconvulsant effects of morphine in mice. Epilepsy Res 2002;48:33-41.

45. Zamanian G, Shayan M, Rahimi N, et al. Interaction of morphine tolerance with pentylenetetrazole-induced seizure threshold in mice: the role of NMDA-receptor/NO pathway. Epilepsy Behav 2020;112:107343.

46. Meskinimood S, Rahimi N, Faghir-Ghanesefat H, Gholami M, Sharifzadeh M, Dehpour AR. Modulatory effect of opioid ligands on status epilepticus and the role of nitric oxide pathway. Epilepsy Behav 2019;101(Pt A): 106563.

47. Rondouin G, Bockaert J, Lerner-Natoli M. L-nitroarginine, an inhibitor of NO synthase, dramatically worsens limbic epilepsy in rats. Neuroreport 1993;4:1187-90.

48. Maggio R, Fumagalli F, Donati E, et al. Inhibition of nitric oxide synthase dramatically potentiates seizures induced by kainic acid and pilocarpine in rats. Brain Res 1995;679:184-7.

49. Wang Q, Theard MA, Pelligrino DA, et al. Nitric oxide (NO) is an endogenous anticonvulsant but not a mediator of the increase in cerebral blood flow accompanying bicuculline-induced seizures in rats. Brain Res 1994; 658:192-8.

50. Del-Bel EA, Oliveira PR, Oliveira JA, Mishra PK, Jobe PC, Garcia-Cairasco N. Anticonvulsant and proconvulsant roles of nitric oxide in experimental epilepsy models. Braz J Med Biol Res 1997;30:971-9.

51. De Sarro G, Di Paola ED, De Sarro A, Vidal MJ. L-arginine potentiates excitatory amino acid-induced seizures elicited in the deep prepiriform cortex. Eur J Pharmacol 1993;230:151-8.

52. Penix LP, Davis W, Subramaniam S. Inhibition of NO synthase increases the severity of kainic acid-induced seizures in rodents. Epilepsy Res 1994;18:177-84.

53. Wink DA, Miranda KM, Espey MG. Cytotoxicity related to oxidative and nitrosative stress by nitric oxide. Exp Biol Med (Maywood) 2001;226:
$621-3$.

54. Umanah GKE, Ghasemi M, Yin X, et al. AMPA receptor surface expression is regulated by S-nitrosylation of thorase and Transnitrosylation of NSF. Cell Rep 2020;33:108329.

55. Ghasemi M, Schachter SC. The NMDA receptor complex as a therapeutic target in epilepsy: a review. Epilepsy Behav 2011;22:617-40.

56. Choi YB, Tenneti L, Le DA, et al. Molecular basis of NMDA receptor-coupled ion channel modulation by S-nitrosylation. Nat Neurosci 2000;3:15-21.

57. Lee $\mathrm{Yl}$, Giovinazzo D, Kang $\mathrm{HC}$, et al. Protein microarray characterization of the S-nitrosoproteome. Mol Cell Proteomics 2014;13:63-72.

58. Lipton $S A$, Choi YB, Takahashi $H$, et al. Cysteine regulation of protein function--as exemplified by NMDA-receptor modulation. Trends Neurosci 2002;25:474-80.

59. Ghasemi M, Shafaroodi $H$, Nazarbeiki $S$, et al. Voltage-dependent calcium channel and NMDA receptor antagonists augment anticonvulsant effects of lithium chloride on pentylenetetrazole-induced clonic seizures in mice. Epilepsy Behav 2010;18:171-8.

60. Tran HQ, Chung YH, Shin EJ, et al. MK-801, but not naloxone, attenuates high-dose dextromethorphan-induced convulsive behavior: possible involvement of the GluN2B receptor. Toxicol App/ Pharmacol 2017;334: 158-166.

61. Reeta Kh, Prabhakar P, Gupta YK. Anticonvulsant activity of the antidepressant drug, tianeptine, against pentylenetetrazole-induced seizures mitigates cognitive impairment in rats. Behav Pharmacol 2016;27: 623-32.

62. O'Neill SK, Bolger GT. Anticonvulsant activity of MK-801 and nimodipine alone and in combination against pentylenetetrazole and strychnine. Pharmacol Biochem Behav 1989;32:595-600.

63. Turski L, Meldrum BS, Cavalheiro EA, et al. Paradoxical anticonvulsant activity of the excitatory amino acid N-methyl-D-aspartate in the rat caudate-putamen. Proc Natl Acad Sci U S A 1987;84:1689-93.

64. Turski WA, Cavalheiro EA, Bortolotto ZA, Ikonomidou C, Turski L. Anticonvulsant activity of $\mathrm{N}$-methyl-D-aspartate in the rat substantia nigra pars compacta. In: Cavalheiro EA, Lehmann J, Turski L, ed. Frontiers in Excitatory Amino Acid Research. New York: Liss, 1988;263-266.

65. Teo SK, Evans MG, Brockman MJ, et al. Safety profile of thalidomide after 53 weeks of oral administration in beagle dogs. Toxicol Sci 2001; 59:160-8.

66. FINCATO M. Potentiating effect of N-phthalyl-glutamic acid imide on barbiturate-induced sleep. Boll Soc Ital Biol Sper 1957;33:872-4.

67. Velišek L. Models of chemically-induced acute seizures. In: Pitkanen A, Schwartzkroin PA, Moshé SL, ed. Models of Seizures and Epilepsy. Amsterdam: Elsevie, 2006;127-152.

68. Socała K, Nieoczym D, Pieróg M, et al. Effect of Tadalafil on seizure threshold and activity of antiepileptic drugs in three acute seizure tests in mice. Neurotox Res 2018;34:333-46. 